\title{
Rhabdomyolysis and Myoglobinuric Nephrosis (Capture Myopathy) in a Striped Dolphin
}

\author{
P. Herráez, ${ }^{1,2}$ E. Sierra, ${ }^{1}$ M. Arbelo, ${ }^{1}$ J. R. Jaber, ${ }^{1}$ A. Espinosa de los Monteros, ${ }^{1}$ and A. Fernández ${ }^{1}$ \\ ${ }^{1}$ Department of Comparative Pathology, Institute of Animal Health, Veterinary College, University of Las Palmas \\ de Gran Canaria, Trasmontana s/n 35413, Arucas Las Palmas, Spain; ${ }^{2}$ Corresponding author (email: pherraez@ \\ dmor.ulpgc.es)
}

ABSTRACT: This report describes delayed myoglobinuric capture myopathy in a striped dolphin (Stenella coeruleoalba) found stranded alive on the coast of Fuerteventura (Canary Islands, Spain). The animal was transported to Gran Canaria where it died 48 hr after stranding. The main lesions consisted of acute rhabdomyolysis affecting both cardiac and skeletal muscles, and myoglobinuric nephrosis. Using immunohistochemistry, degenerate myofibers with depletion of myoglobin, and an intracytoplasmatic immunoreaction for fibrinogen were observed. Orange-red pigmented casts in renal tubular lumens were strongly immunolabeled for myoglobin. To our knowledge, this is the first pathologic description of capture myopathy with myoglobinuric nephrosis in stranded cetaceans. Stress, exertion, trauma, and crush injury caused during the stranding, restraint, and transportation were the main causes of rhabdomyolysis in this case.

Key words: Capture myopathy, cetaceans, dolphin, fibrinogen, myoglobin, myoglobinuric nephrosis, rhabdomyolysis, Stenella coeruleoalba.

Capture myopathy $(\mathrm{CM})$ is a metabolic muscle disease described in wild mammals associated with stress of capture, restraint, and transportation. Four clinical syndromes are associated with CM: capture shock, ruptured muscle, ataxic myoglobinuric, and delayed peracute (Spraker, 1993). Ataxic myoglobinuric and delayed peracute syndromes occur most commonly, and pathologic findings include mild to moderate rhabdomyolysis affecting the cardiac and skeletal muscle. Moreover, in ataxic myoglobinuric syndrome, renal lesions have been described consisting of moderate to severe tubular necrosis with intratubular protein casts; the urinary bladder usually contains a small amount of brownish urine (Spraker, 1993).

Stress, exertion, and crush injury are well-documented causes of rhabdomyoly- sis, but other causes, such as procedures that involve long periods of restraint, struggling from unnatural positioning, or lengthy pursuit during capture, are also major factors in the development of CM in wildlife (Williams and Thorne, 1996).

Capture myopathy has been documented in ungulates, carnivores, rodents, primates, marsupials, pinnipeds, and birds. Previous studies in cetaceans have demonstrated hematologic changes indicative of activation of the hypothalamicpituitary-adrenal axis and muscle damage in response to capture stress (St. Aubin and Geraci, 1989). In 1978, Colgrove (1978) reported a suspected transportation-associated myopathy in a bottle-nosed dolphin based on increased serum enzyme activities. However, descriptions of the pathologic features of myoglobinuric CM in stranded dolphins appear to be lacking.

We describe the gross and histopathologic features of CM in an active stranded striped dolphin (Stenella coeruleoalba). Acute rhabdomyolysis affecting both cardiac and skeletal muscles and myoglobinuric nephrosis were the distinctive lesions. Stress, trauma, and excessive activity as well as prolonged muscle compression during the stranding, restraint, and transportation are considered to be the most probable causes of muscular damage.

On 20 November 2004, a live adult male striped dolphin was found stranded on the coast of Fuerteventura (Canary Islands, Spain, $\left.28^{\circ} 44^{\prime} \mathrm{N}, 13^{\circ} 52^{\prime} \mathrm{W}\right)$. The animal was in good body condition and kept in a private swimming pool until transported by car and helicopter to the Wildlife Rescue Center facilities in Gran Canaria. The animal died $48 \mathrm{hr}$ later, without 
specific clinical signs; a complete necropsy was done within $2 \mathrm{hr}$ of death at the Veterinary College in Gran Canaria.

At necropsy, the heart had multiple, locally extensive, pale, whitish subendocardial areas in the ventricular myocardium. Kidneys were deeply dark, whereas the urinary bladder was empty. No gross lesions were detected in skeletal muscles. Other gross lesions included a rostral mandibular fracture with loss of soft tissue from the nose caused during the stranding, mild multifocal granulomatous verminous pneumonia, moderate peritoneal parasitism (Monorigma grimaldi), small intestine catharral enteritis, and multifocal parasitic cholangitis (Campula $\mathrm{sp}$ ).

Selected tissue samples were collected, fixed in $10 \%$ neutral buffered formalin, and embedded in paraffin wax, and sections $(4 \mu \mathrm{m})$ were stained with hematoxylin and eosin, PTAH, and Masson's trichrome techniques. In addition, immunohistochemical examination was performed on cardiac, skeletal muscle, and renal tissue sections using the avidinbiotin-peroxidase method (Vector Laboratories, Burlingame, California, USA). Primary antibodies consisted of rabbit antihuman myoglobin diluted to 1:2,000, rabbit antihuman fibrinogen diluted to 1:200, and rabbit antihuman Heat Shock Protein 70 (HSP70) diluted to 1:100 (Dako, Glostrup, Denmark). Tissue sections in which the primary antibodies were replaced by phosphate-buffered saline or nonimmune serum (rabbit or mouse) were used as negative controls. Kidney and skeletal and cardiac muscle tissue sections from horses with exertional rhabdomyolysis were used as positive controls.

Histopathologic examination of the myocardium revealed multiple, locally extensive areas of acute myocyte degeneration. Myocyte changes consisted of cytoplasmic eosinophilia, loss of cross striations, cytoplasmic fragmentation, myocytolysis, and nuclear condensation (Fig. 1). Limited to the immediate subendocardial regions, scattered myocytes had dense, hypereosi-

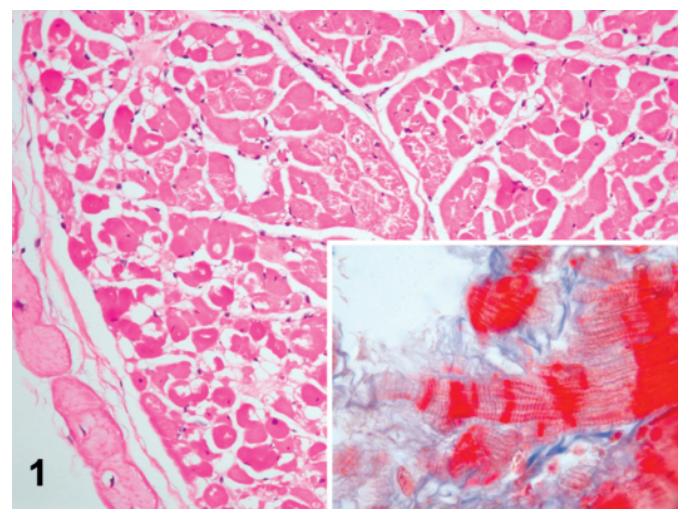

Figure 1. Heart: Myocytes have acute degenerative changes characterized by cytoplasmic fragmentation and myocytolysis (hematoxylin and eosin method). Inset: Subendocardial myocytes with contraction band necrosis (Masson's trichrome method).

nophilic bands running transversely across the cytoplasm (Fig. 1, inset), which were indicative of contraction band necrosis. Immunohistochemically, degenerate myocytes had depletion of myoglobin (Fig. 2a) and an intense cytoplasmic immunoreaction with antifibrinogen antibody (Fig. 2b). A strong, homogeneous immunoreaction with antimyoglobin antibody was detected in the intravascular plasma of medium- to small-size vessels in the affected areas. Similar degenerative changes were de-

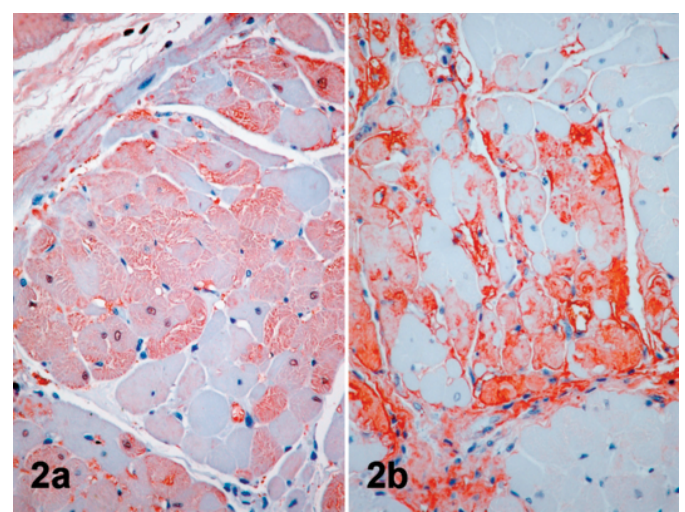

Figure 2. Heart: (a) Depletion of myoglobin in degenerate myocytes is demonstrated by decreased immunoreaction for myoglobin. (b) Strong intracytoplasmatic immunoreaction for fibrinogen in affected myocytes (avidin-biotin-peroxidase complex method with 3-amino-9 ethylcabazole as chromagen). 


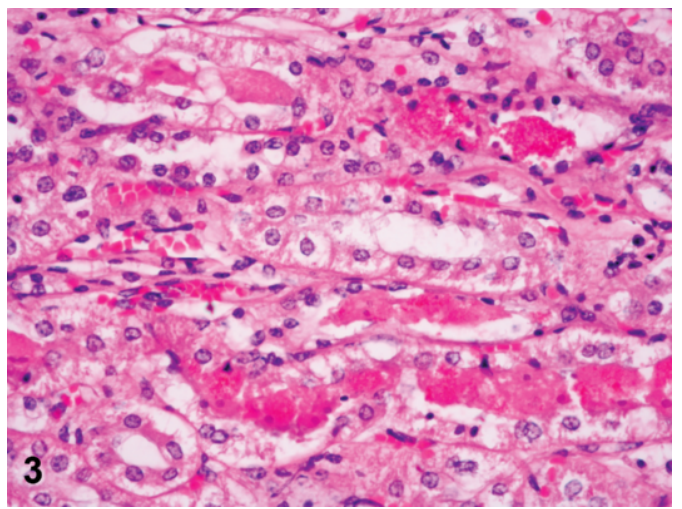

Figure 3. Kidney: Intratubular pigmented casts associated with tubular epithelial cells swelling (hematoxylin and eosin method).

tected in skeletal muscles including depletion of myoglobin and a cytoplasmic immunoreaction for fibrinogen. Renal lesions consisted of extensive swelling of renal tubular cells and pigmented orangered, homogeneous intratubular casts (Fig. 3). Granules, droplets, and casts in Bowman's space, tubular lumens, and cytoplasm of degenerate tubular cells were strongly labeled by the antimyoglobin antibody (Fig. 4a). In addition, some of these degenerate cells had a granular cytoplasmic immunoreaction for HSP70 (Fig. 4b). No other lesions were observed.

Capture myopathy is an acute disease characterized by rhabdomyolysis and renal tubular necrosis that occurs in wild animals hours or days following a chase, a struggle, or transport. No specific clinical signs indicative of rhabdomyolysis were detected in this case, and the animal died $48 \mathrm{hr}$ after the stranding. Although CM can cause depression, hyperthermia, ataxia, torticollis, and myoglobinuria, in many cases, the disease is asymptomatic and only laboratory abnormalities are detected (Spraker, 1993). Myoglobinuria is the central element in the development of rhabdomyolysis-induced acute renal failure, and it is frequently considered to be the first clinical sign indicator of myoglobinuric nephrosis. However, the gross diagnosis of $\mathrm{CM}$ in cetaceans can

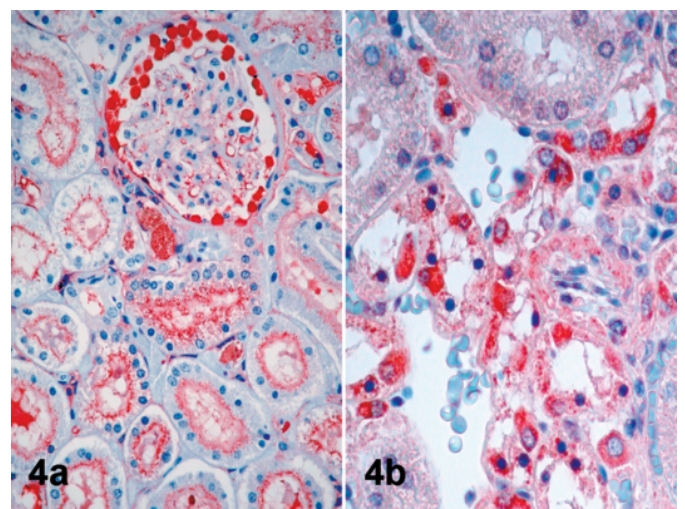

Figure 4. Kidney: (a) Immunoreaction for myoglobin in the granules and casts in the Bowman space and tubular lumen. The apical cytoplasm of degenerate tubular cells shows immunoreaction for myoglobin. (b) Some of damaged tubular cells show immunoreaction for anti-HSP70 antibody (avidinbiotin-peroxidase complex method with XXX as chromagen).

be complicated because the urinary bladder of cetaceans usually is devoid of urine at necropsy, they urinate in the aquatic environment, making visualization of the urine difficult, and the kidneys usually are deeply dark secondary to vascular compromise.

Myocardial lesions consisted of multifocal degenerative changes and subendocardial areas of contraction band necrosis. Contraction band necrosis develops after transient ischemia and reperfusion, and it is the characteristic myocardial lesion associated with elevated concentrations of endogenous catecholamines (Jiang and Downing, 1990). This lesion is recognized in wide clinical circumstances, including stress and trauma, and it has been implicated as a mechanism of sudden death in these situations. Similar cardiac lesions described in stranded cetaceans have been attributed to physiologic or psychologic stress related to stranding, injury, or disease (Turnbull and Cowan, 1998). In addition, the immunohistochemical study revealed depletion of myoglobin and cytoplasmic immunoreaction for fibrinogen, which could indicate hypoxic cellular injury. Fibrinogen and myoglobin 
have been used as morphologic parameters of early myocardial ischemia. Experimental studies have demonstrated that acute ischemia produces early myocardial cell membrane rupture, causing depletion of cytoplasmic myoglobin and deposition of plasma proteins such as fibrinogen in myocytes (Xiaohong et al., 2002).

No gross lesions were observed in skeletal muscles, and tissue samples were routinely obtained from superficial portions of the mulitifindus and longissimus muscles just caudal to the dorsal fin. Previous studies have not described pathologic changes in locomotory muscles in dolphins that died during fishing operations (Cowan and Walker, 1979), which has been interpreted as the usual absence of muscle lesions in the delayed-peracute capture myopathy syndrome (Curry, 1999). However, histologic and immunohistochemical examination of skeletal muscles in our study revealed similar lesions to those described in the myocardium, indicating ischemic damage. Exertion, trauma, and crush muscular injury during the stranding and transportation are considered to be the main causes of skeletal muscular lysis in our case. The mechanisms underlying exercise-induced rhabdomyolysis remain unclear, but hypokalemia and ischemia appear to be involved (Guis et al., 2005). In traumatic and crushinduced rhabdomyolysis, the muscles are initially compressed and ischemic, and muscle dysfunction starts to develop only when the animals are evacuated, causing reperfusion of the damaged muscles (reperfusion injury) (Odeh, 1991). A comparable situation could have happened in our case when the animal was reintroduced into an aquatic medium after the active stranding and transportation.

Regardless of the initiating mechanism, rhabdomyolysis results in the release of muscular cell components into circulation, mainly myoglobin. Myoglobin is an important storage site for oxygen in the muscles of diving marine mammals, and their high muscular concentration is con- sidered to be one of their most important adaptations. However, myoglobin in the locomotory muscles is not homogeneously distributed. Myoglobin is found in greater concentrations in the epaxial than in the hypaxial muscles, with the highest concentrations located in deeper portions close to vertebrae and in the most caudal region closest to the flukes, which produce thrust during swimming (Dolar et al., 1999). In addition, the rectus abdominis muscle, which is a nonaxial muscle that originates at the pubic symphysis and inserts into the sternum and costal cartilages, has a myoglobin concentration comparable to the epaxial locomotory muscle (Dolar et al., 1999), and it could have suffered traumatic and compressive injury during the stranding and posterior transportation, contributing to the development of the myoglobinemia in our case. Data related to myoglobin distribution in cetaceans probably should be used to target those groups of muscles likely to be most susceptible to exertional rhabdomyolysis and its consequences for examination.

Rhabdomyolysis can cause the death via several mechanisms, including heart failure, volumen depletion, metabolic acidosis, hypocalcemia, hyperphosphatemia, and disseminated intravascular coagulation. Acute renal failure (ARF) is the most important and frequent complication in rhabdomyolysis, mainly in animals with large muscle masses (Guis et al., 2005). Renal lesions in this case were similar to those described in other mammalian myoglobinuric nephrosis. Cowan and Curry (2002) reported on a series of dolphins with lesions related to capture and chase during fishery operations; all animals studied had acute necrosis of renal tubules attributed to ischemia but none had intratubular myoglobinuric casts. In this case, immunohistochemistry was useful for demonstrating myoglobin as the origin of the intratubular casts and identifying myoglobinuric-related tubular degeneration. The main pathophysiologic mech- 
anisms of myoglobinuric ARF include renal vasoconstriction, intraluminal cast formation, and direct heme protein-induced cytotoxicity (Zager, 1996). In our study, some of the degenerate epithelial tubular cells were immunohistochemically reactive for HSP70. Previous studies have indicated that the immunohistochemical staining of myoglobin and oxidative injuryrelated markers, including HSP70, offers important information for the diagnosis of myoglobinuric-related ARF (Ishigami et al., 2003).

In conclusion, this study showed that CM should be included in the differential diagnostic of delayed death in active stranded cetaceans, the importance of an exhaustive examination of skeletal muscle in order to find degenerative changes, and the usefulness of the inmunohistochemical demonstration of fibrinogen, myoglobin, and HSP70 as markers of early ischemic muscle damage and myoglobinuric nephrosis in dolphins.

\section{LITERATURE CITED}

Colgrove, G. S. 1978. Suspected transportationassociated myopathy in a dolphin. Journal of American Veterinary Medical Association 173: 1121-1123.

Cowan, D. F., and B. E. Curry. 2002. Histopathological assessment of dolphins necropsied onboard vessels in the eastern tropical Pacific tuna fishery. Southwest Fisheries Science Center, National Marine Fisheries Service, National Oceanographic and Atmospheric Administration (NOAA) Administrative Report No. LJ-02-24C, Santa Cruz, California, 31 pp.

—, AND W. A. WALKER. 1979. Disease factors in Stenella attenuata and Stenella longirostris taken in the eastern tropical Pacific Yellowfin Tuna Purse Seine Fishery. Southwest Fisheries Science Center, National Marine Fisheries Service, National Oceanographic and Atmospheric Administration (NOAA) Administrative Report No. LJ-79-32C, Santa Cruz, California, 21 pp.

Curry, B. E. 1999. Stress in mammals: The potential influence of fishery-induced stress on dolphins in the eastern tropical Pacific Ocean. Southwest
Fisheries Science Center, National Marine Fisheries Service, National Oceanographic and Atmospheric Administration (NOAA) Administrative Report No. NOAA-TM-NMFS-SWFSC, Santa Cruz, California, 260 pp.

Dolar, M. L., P. Suarez, P. J. Pnganis, and G. L. Kooyman. 1999. Myoglobin in pelagic small cetaceans. Journal of Experimental Biology 202: 227-236.

Guis, S., J. P. Mattei, P. Cozzone, and D. Bendahan. 2005. Pathophysiology and clinical presentations of rhabdomyolysis. Joint Bone Spine 72: 382391.

Ishigami, A., I. Tokunaga, T. Gotohda, and S. Kubo. 2003. Immunohistochemical study of myoglobin and oxidative injury-related markers in the kidney of methamphetamine abusers. Legal Medicine 5: 42-48.

Jiang, J. P., ANd S. E. Downing. 1990. Catecholamine cardiomyopathy: Review and analysis of pathogenetic mechanisms. Yale Journal of Biology and Medicine 63: 581-591.

Open, M. 1991. The role of reperfusion-induced injury in the pathogenesis of the crush syndrome. New England Journal of Medicine 324: 1417-1422.

SPRAKER, T. R. 1993. Stress and capture myopathy in artiodactyls. In Zoo and Wild Animal Medicine, Current therapy 3, M. E. Fowler (ed.). W.B. Saunders Company, Philadelphia, Pennsylvania, pp. $481-488$.

St. Aubin, D. J., and J. R. Geraci. 1989. Adaptative changes in hematologic and plasma chemical constituents in captive beluga whales, Delphinapterus leucas. Canadian Journal of Fisheries and Aquatic Sciences 46: 796-803.

Turnbull, B. S., AND D. F. Cowan. 1998. Myocardial contraction band necrosis in stranded cetaceans. Journal of Comparative Pathology 118: 317-327.

Williams, E. S., and E. T. Thorne. 1996. Exertional myopathy (capture myopathy). In Noninfectious diseases of wildlife. 2nd Edition, A. Fairbrother, L. N. Locke and G. L. Hoff (eds.). Iowa State University Press, Ames, Iowa, pp. 181-193.

Xiaohong, Z., C. Xiaorui, H. Jun, and Q. Qisheng. 2002. The contrast of immunohistochemical studies of myocardial fibrinogen and myoglobin in early myocardial ischemia in rats. Legal Medicine 4: 47-51.

ZAGER, R. A. 1996. Rhabdomyolysis and myohemoglobinuric acute renal failure. Kidney International 49: 314-326.

Received for publication 20 July 2006. 